\title{
The protein decade
}

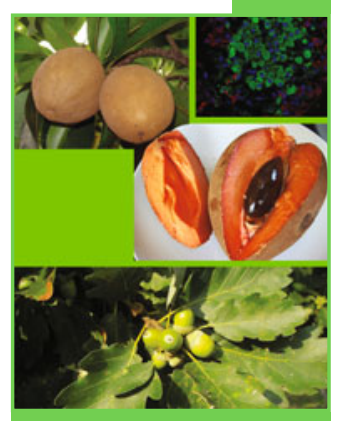

Correspondence to:

Andrea Poli

poli@nutrition-foundation.it

(C) Springer - CEC Editore 2014

The scientific community's interest in proteins and peptides in nutrition is continuously increasing. This is a genuine interest, well beyond hyper-proteic diets, their potential role in weight management, the increased requirements of the elderly, and the often-useless debates on vegetable vs. animal protein, their higher or lower content of essential amino acids and their contribution to cardiovascular risk (see e.g. the China study).

The many variants that make up protein's primary structure, i.e. the amino acid sequence of these macromolecules, make them very flexible in terms of both secondary and tertiary structures. This has functional consequences, because longer and shorter peptide sequences can activate cellular receptors, similar to the process in endogenous molecules. Therefore, the activities of enzymes or biological pathways can be influenced by small proteins.

We are now realising that these peptides, frequently generated during hydrolysis of dietary protein, can be absorbed and circulate even if their molecular weight is quite high. The foremost example is milk protein, whose tripeptides have, for example, ACE- inhibitory activities which lead to small, but significant, hypotensive actions.

This issue of NutraFoods features a review by Miguel E. Oseguera et al. that addresses the role of dietary peptides in glycaemic metabolic control. In particular, peptides of various origins are capable of inhibiting the activity of DPP-IV (which degrades and inactivates GLP-1, an insulinotropic incretin). This is the same mechanism of action as in several synthetic drugs found in the pharmaceutical market. The authors critically propose the incorporation of such peptides (or their proteic precursors) in daily foods, which might lead to important preventive or therapeutic outcomes. In the future, we might be able to identify specific foods (or their proteic components) that - after digestion and interaction with the microbiota - could generate peptides with remarkable biological activities. Indeed, similar things happen every day in our intestine; by exploiting our knowledge of such biological processes we can apply them to prevention and therapy. The too-oft-quoted Hippocrates line "Let food be your medicine" might evolve into "Let food protein be your medicine". 